\section{A TIME OF CELEBRATION}

South Africa breathed an audible sigh of relief last month when our cabinet finally agreed to a national antiretroviral rollout programme. Apparently and correctly there is a rea sense of urgency to translate this into numbers of people on treatment as soon as possible. This is in keeping with the World Health Organisation's target, expressed on 1 December this year, of 3 million people on antiretroviral treatment by 2005 . The South African approval for rollout has been dependent on the provision of a plan that would be acceptable to government. Happily this plan, formulated in record time by a task force headed up by Dr Tony Mbewu and with substantial input from the Clinton Foundation experts, required only a readdressing of infrastructure support before being pronounced adequate. Such a national plan, which has been based on WHO treatment guidelines, means that there will be conformity and consistency throughout the country and streamlines a number of logistic issues such as staff training, drug procurement, laboratory needs and evaluation procedures. This conformity is ideal as long as the implementation plan is based on perfect guidelines. We all know, however, that guidelines quickly become outdated and irrelevant unless they undergo revision as new data are gathered. However, there is a real danger that as one plan is adopted the input from a diversity of protocols and projects will be lost. In South Africa, as we put our energy into the rollout of a single plan, we should continue to encourage and fund pilot sites that will test new or different strategies, such as different drug regimens, adherence strategies, monitoring schedules, or even treatment strategies such as intermittent therapy. Logistics such as methods of drug and data management may also be varied. With adequate evaluation and assessment, lessons learnt from these pilot sites should then be used to modify the national programme appropriately and ensure that our practice does not become outdated and ineffectual. Critical to the success of the national programme is an evaluation process that will quickly identify shortcomings in the programme and enable modifications to occur. It will also need to respond dynamically to changes in the epidemic such as changes in drug sensitivity, population targets, etc. Evaluation should also be sensitive enough to allow quality assessment from site to site, again enabling real-time modification and improvement. An awesome task, but one that must happen and must succeed.
How appropriate in this time of celebration that Zackie and TAC have been nominated for a Nobel prize! We are immensely proud of this activist organisation. In contrast, one remembers how destructive and obstructive the early US and European activist community was. They appeared to be passionately opposed to everyone, especially the medical community. TAC has worked with the medical community towards a common goal of care and treatment for the HIV-infected. I guess this has been one good outcome of a perceived obstruction from government - it has united activists and the medical community in a common mission. TAC, together with the AIDS Law Project, has rationally and cleverly used the excellent laws and constitution of this country to move this agenda forward, both in terms of government policy and in relation to the pharmaceutical industry. The recent settlement with pharmaceutical companies on the Competitions Law in this country is yet another example of putting good laws to constructive use and has resulted in benefit to the country as a whole.

Speaking of missions, wasn't it great to be united in music at the 46664 concert recently held at Greenpoint stadium in Cape Town? How spectacular to see South Africans really caring about the plight of the HIV-infected, and to see some of those old (and a few new) rockers giving up time and energy to make a difference. It wasn't about the quality of the music, but the quality of feeling.

As we look back on the past year in South Africa, it is as usual astounding to note the water that has flowed under the proverbial bridge. I am reminded of a line in a one-person play that I saw in New York some years ago in which it was said that more happened in a day in Israel than happened in a year in Norway! South Africa in its young democracy is a bit like the former - but to the HIV community the fact that treatment is on the cards is undoubtedly the most exciting. Roll on 2004, when we will actually see the treatment happen and lives and communities be changed as a result.

We wish all of our readers a most wonderful holiday season, and thank the staff at SAMA, Des and the staff at the HIV Clinicians Society office for all their hard work, and also all the contributors to the journals this year - we look forward to a torrent of articles, papers and contributions in the new year! Keep them coming!

LINDA-GAIL BEKKER

Managing Editor 